\title{
Contaminação de lâminas de silício por metais durante a imersão em solução diluída de ácido fluorídrico ou de água deionizada contendo cloretos metálicos
}

\section{Contamination of silicon wafers by metals during immersion in diluted hydrofluoric acid or deionized water containing metal chlorides}

Elvis Melo Oliveira ${ }^{1}$, Sebastião Gomes dos Santos Filho'

\section{RESUMO}

A contaminação metálica em semicondutores e, especialmente em silício, é uma causa bem conhecida de falhas em dispositivos MOS. Assim, compreender os mecanismos de adsorção de metais na superfície do silício é de extrema importância e foi objetivo deste trabalho. Contaminou-se intencionalmente a superfície de lâminas de silício com 100 mm de diâmetro usando solucão diluída e contaminada de ácido fluorídrico para testes de adsorção e água deionizada contendo cloretos metálicos como branco. As concentrações superficiais de contaminantes residuais tais como $\mathrm{Ca}, \mathrm{Fe}, \mathrm{Ni}, \mathrm{Co}, \mathrm{Cu}$ e $\mathrm{Cl}$ foram determinadas após a imersão. No branco, tanto o cloreto como o seu metal correspondente foram detectados na superfície do silício e aparentemente a relação entre as concentrações superficiais de cloreto e metal não seguiu a estequiometria original do cloreto metálico empregado como fonte de contaminação intencional. Por outro lado, depois da imersão em solução diluída de ácido fluorídrico: i) ferro e cloreto são observados na superfície do silício, possivelmente devido à complexação do ferro com o cloreto, ii) traços de Ca e Co são observados na superfície do silício, possivelmente devido à precipitação/complexação com fluoreto e cloreto, respectivamente e iii) foi observada a ocorrência de deposição espontânea de cobre. Um modelo qualitativo utilizando as solubilidades dos sais e o potencial eletroquímico pode explicar tais resultados.

Palavras-chave: Cloretos metálicos; Caracterização de superfícies; Contaminação de superfícies.

\begin{abstract}
Metal contamination in semiconductors, and specially on silicon, is a well-known cause of failures in MOS devices. Therefore, understanding mechanisms of metal adsorption on silicon surface is quite relevant and for this reason this work performed an intentional contamination of silicon wafers, $100 \mathrm{~mm}$ in diameter. It was used immersion in contaminated diluted hydrofluoric solution for adsorption tests and in deionized water containing metal chlorides as blank. Surface concentrations of residual contaminants such as $\mathrm{Ca}, \mathrm{Fe}, \mathrm{Ni}, \mathrm{Co}, \mathrm{Cu}$ e $\mathrm{Cl}$ were characterized after immersion. In the blank, both chloride and its corresponding metal were detected on the surface of silicon and the relation between surface concentrations of chloride and metal does not follow the original stoichiometry of the metal chloride used as a source of intentional contamination. On the other hand, after immersion in diluted hydrofluoric solution: i) iron and chloride are observed on the silicon surface, possibly due to complexation of iron with chloride, ii) traces of $\mathrm{Ca}$ and $\mathrm{Co}$ are observed on the silicon surface, possibly due to precipitation/complexation with fluoride and chloride, respectively, and iii) spontaneous deposition of copper was observed to occur. A qualitative model considering salt solubility and electrochemical potential can explain the results.
\end{abstract}

Keywords: Metal chlorides; Surface characterization; Surface contamination. 


\section{INTRODUÇÃO}

$\mathrm{Na}$ indústria de semicondutores, é comumente aceito que o controle rigoroso sobre a distribuição das impurezas metálicas superficiais em lâminas de silício é um parâmetro crítico para garantir a produção com alto rendimento de dispositivos baseados na tecnologia do silício(1). Um controle efetivo necessita de um minucioso entendimento das reações e precipitações metálicas, uma vez que estas determinam a concentração metálica residual homogeneamente aderida na superfície de lâminas de silício ${ }^{(1,2)}$. Circuitos em escala de integração muito ampla (ULSI: Ultra Large Scale Integration) são muito mais susceptíveis à contaminação metálica que seus predecessores (LSI: Large scale Integration and VLSI: Very Large Scale Integration), devido às geometrias muito pequenas, às junções rasas, e às grandes áreas das pastilhas de circuito integrado (Chip) ${ }^{(2)}$. Portanto, é fundamental manter essa contaminação sob controle durante o processamento dos circuitos integrados, os quais são muito sensíveis a traços de impurezas. Quando metais difundem na rede do silício, são introduzidos níveis eletrônicos ao longo da banda proibida anteriormente inexistentes e que mais tarde podem atuar no aumento da corrente de fuga em junções p-n e na redução do tempo de vida de geração dos portadores de carga minoritários.

Níveis de contaminação residuais na escala de $1,0 \times 10^{11}$ à $1,0 \times 10^{13}$ atomos $/ \mathrm{cm}^{2}$, dependendo do tipo de chip, têm geralmente sido permitidos nos processos de manufatura ${ }^{(3,4)}$. Para os atuais chips com dispositivos menores do que $0,1 \mu \mathrm{m}$, um nível de contaminação abaixo do que valores da ordem de $10^{10}$ átomos $/ \mathrm{cm}^{2}$ tornou-se imperativo ${ }^{(5,6)}$.

Apesar de todo o avanço tecnológico na área de circuitos integrados, ainda persistem os principais focos de contaminação durante:

a. o crescimento do cristal;

b. o corte e o polimento das lâminas e

c. a fabricação dos dispositivos.

Os dispositivos de silício são influenciados pela presença de diversos tipos de contaminantes tais como elementos da família 13 e 15 da tabela periódica os quais promovem níveis rasos na faixa de energia proibida ou os elementos da família 1 e 2 (alcalinos e alcalinos terrosos) os quais degradam fortemente a qualidade do óxido de silício de porta em dispositivos MOS. Portanto, a contaminação por metais de transição continua sendo uma das principais preocupações na tecnologia moderna dos semicondutores ${ }^{(6)}$. As impurezas contaminantes mais comuns pertencem ao grupo dos metais de transição que podem ocupar posições intersticiais ou substitucionais ${ }^{(7)}$. Estes átomos de impurezas são denominados defeitos extrínsecos e como eles geralmente têm um tamanho diferente dos átomos da rede, eles introduzem uma perturbação, mesmo quando são substitucionais. A rede de silício, por ser menos compacta que outras redes, por exemplo, que uma rede simples do tipo fcc, tem mais vazios e isso justifica a facilidade com que aparecem os átomos intersticiais bem como a existência de uma concentração de equilíbrio de vacâncias bem menor que a concentração típica em ligas metálicas. Os metais localizados na tabela periódica entre escândio e ferro são impurezas intersticiais e cresce a probabilidade dos átomos ocuparem posições substitucionais para o caso do cobalto, níquel e cobre, até chegar ao zinco, que ocupa posição substitucional exclusivamente.

$\mathrm{Co}, \mathrm{Ni}$ e $\mathrm{Cu}$ difundem muito rapidamente mesmo à temperatura ambiente no silício, não são estáveis nos estados intersticiais e precipitam ou formam complexos ou aglomerados durante tratamentos térmicos ${ }^{(8,9)}$. Cobre e níquel podem causar ruptura nos óxidos de silício empregados em portas $\operatorname{MOS}^{(9,11,12)}$ ao passo que Ferro-Boro tem um efeito prejudicial na eficiência de células solares em concentração ao redor de $10^{13} \mathrm{~cm}^{-3(7,8,9)}$ e mesmo na escala ${ }^{(13)}$ de $10^{11} \mathrm{~cm}^{-3}$. O ferro é uma das mais importantes impurezas já que é o principal constituinte dos muitos materiais tecnicamente importantes e, portanto, é uma impureza muito comum $^{(10,14)}$. Ambos os casos, difusão do cobre e ferro, são influenciados pela existência de defeitos na rede, tanto pontuais, como presença de discordâncias, fronteiras de grão entre outros.

Porém, para cada tipo de metal existe uma concentração específica a partir da qual a degradação se faz presente. Níveis de contaminação de superfície de $5 \times 10^{12} \mathrm{~cm}^{-2}$ foram mostrados que prejudicam o óxido de porta ${ }^{(15-22)}$. Ambas as conclusões derivam dos diferentes valores da secção de captura dos minoritários (elétrons no Si tipo p, lacunas no Si tipo n) dos diferentes metais. A seção transversal de captura para cargas majoritárias e minoritárias de nível profundo, induzidas por $\mathrm{Co}, \mathrm{Ni}$ e $\mathrm{Cu}$, os quais determinam esse comportamento de recombinação, são incertas ou desconhecidas ${ }^{(9)}$. Desta forma, torna-se necessário evitar, dentro do possível, a contaminação, para obter dispositivos de alta eficiência.

Assim como o cobre, o níquel não permanece em interstícios na temperatura ambiente e precipita rapidamente após etapas de resfriamento. Sabe-se que o níquel forma precipitados primariamente na superfície após um resfriamento lento de lâminas $\mathrm{Cz}$ ou Fz. O cobalto é o mais leve e de mais rápida difusão dos metais de transição. A solubilidade do cobalto é menor que a do cobre e a do níquel e está próxima a solubilidade do ferro e do manganês ${ }^{(15)}$.

As estratégias habitualmente seguidas para evitar a contaminação durante os processos de fabricação de circuitos integrados compreendem realizar processos de limpeza que eliminem as fontes de contaminação superficiais e incorporar técnicas denominadas de armadilhamento de impurezas (gettering). Metais mais nobres, como o cobre, tendem a ser adsorvidos e reduzidos sobre as lâminas de silício ${ }^{(16-31)}$. Os processos de gettering produzem a extração e/ou neutralização de impurezas contaminantes na área ativa do dispositivo, realizada durante o crescimento do cristal ou em passos subseqüentes da fabricação de dispositivos eletrônicos.

Os metais de forma geral têm grande probabilidade de serem capturados nos defeitos extensos. A presença dos defeitos extensos 
decorados por metais pode prejudicar o funcionamento de um dispositivo, pois causam grandes correntes de fuga e diminuem a tensão de ruptura. Hoff, et. al. demonstraram que a exposição de óxidos finos a soluções contendo água ultra pura e metais de transição tais como $\mathrm{Co}, \mathrm{Cu}, \mathrm{Ni}$ e $\mathrm{Fe}$ produzem dramáticas degradações nas propriedades dielétricas de tais óxidos. O Cu tem o maior impacto sobre a qualidade dielétrica seguido por Co, em seguida, Fe e, finalmente $\mathrm{Ni}$, em ordem de gravidade $^{(32)}$.

Em trabalho anterior ${ }^{(20)}$, empregaram-se sulfatos metálicos no estudo da contaminação intencional de lâminas de silício por soluções diluídas de ácido fluorídrico. Naquela oportunidade, foi estabelecido um mecanismo eletroquímico que descrevia a contaminação superficial por metal e enxofre. No presente artigo utiliza-se contaminação intencional das soluções empregando cloretos metálicos para analisar os possíveis mecanismos de contaminação.

\section{PROCEDIMENTOS EXPERIMENTAIS}

\section{Preparação das amostras para estudo de contaminação intencional}

O preparo das soluções para estudo de contaminação intencional por cloretos metálicos utilizou sempre água deionizada filtrada (ADIF) com resistividade de $18 \mathrm{M} \Omega . \mathrm{cm}$ e reagentes grau MOS (HF) ou PA (cloretos metálicos) de pureza. O procedimento de testes fez uso de duas soluções distintas:

a. solução aquosa de cada cloreto metálico, de tal forma que um contaminante específico $\left(\mathrm{Ca}^{2+}, \mathrm{Fe}^{2+}, \mathrm{Co}^{2+}, \mathrm{Ni}^{2+}\right.$ ou $\mathrm{Cu}^{2+}$ ) estivesse dissolvido em concentrações variando na faixa de 100 a 500 ppm. Essa solução foi utilizada como branco e denominada (ADIFc);

b. solução diluída de ácido fluorídrico, preparada pela mistura do ácido fluorídrico concentrado (49\%) à solução aquosa descrita em (a), tal que a diluição ficasse na faixa de $0,3125 \mathrm{M}$ a $2,5 \mathrm{M}$.

Essa solução foi denominada D-HF Todas as soluções foram sempre preparadas em béqueres de Teflon de $2000 \mathrm{ml}$. O uso de ácido fluorídrico justifica-se por ser a última etapa de limpeza no processamento inicial de lâmina de silício ${ }^{(20)}$

Foram empregadas lâminas de silício com $100 \mathrm{~mm}$ de diâmetro, resistividade de 10 a $20 \Omega \mathrm{cm}$ e com orientação $<100>$. Estas lâminas sempre apresentaram um baixo nível de contaminação metálica superficial inicial $\left(<1 \times 10^{11}\right.$ átomos $\left./ \mathrm{cm}^{2}\right)$ e um óxido nativo com uma espessura estimada de $3 \mathrm{~nm}$.

Foram preparados vários pares de lâminas, ao menos um par para cada cloreto metálico utilizado como fonte de contaminação. Primeiramente, uma das lâminas de um dado par é imersa no branco (ADIFc) de um cloreto metálico específico, mantendo-se a solução em regime de agitação para minimizar qualquer efeito de depleção de metal junto à superfície da lâmina e utilizando-se a própria lâmina como agitador de solução. Após um intervalo de 120 s, cessa-se a agitação e a lâmina é então retirada da solução e seca em nitrogênio. Em seguida, a outra lâmina do par é imersa na solução ácida diluída (D-HF), também em regime de agitação, utilizando-se a própria lâmina como agitador de solução. Após o mesmo intervalo de tempo, a lâmina foi retirada da solução e uma vez que ela saiu seca, não foi realizado nenhum processo de secagem adicional.

\section{Técnicas de medida da concentração superficial de metais}

Após a imersão no branco (ADIFc) ou em solução aquosa de ácido fluorídrico diluído (D-HF), as lâminas com 100 mm de diâmetro foram analisadas por fluorescência de raios X na condição de reflexão total (TXRF: total reflection X-ray fluorescence) ${ }^{(16,17,21)}$ a fim de se obter a concentração superficial de contaminantes tais como $\mathrm{Ca}, \mathrm{Fe}, \mathrm{Ni}, \mathrm{Co}, \mathrm{Cu}, \mathrm{Cl}$ os quais podem permanecer na superfície das lâminas. Estas medidas de TXRF permitem obter as concentrações superficiais destes contaminantes em sítios com uma área típica de $1 \mathrm{~cm}^{2(16)}$. Foram medidos sistematicamente três sítios em cada lâmina e em seguida foram obtidos os valores médios das concentrações superficiais para cada contaminante nestes três sítios.

\section{RESULTADOS E DISCUSSÃO}

A Tabela 1 mostra as melhores respostas obtidas para a adsorção, indicando as concentrações superficiais de cloreto e de seu metal correspondente depois da imersão da lâmina numa condição de agitação durante 120s em ADIFc ou D-HF, contaminados com 500 ppm de cada um dos cloretos metálicos descritos nos procedimentos experimentais. A Figura 1 ilustra o espectro TXRF obtido para amostra que sofreu imersão no branco (ADIFc) enquanto a Figura 2 ilustra também o espectro TXRF para a mesma solução de cloreto de cobalto, agora com o uso de ácido fluorídrico. Comparando as duas figuras, observase claramente a diminuição substancial da concentração de cobalto e de cloreto na superfície da lâmina de silício.

Analisando-se os resultados de imersão em ADIFc na Tabela 1, podemos concluir que tanto o cloreto como o seu metal correspondente em solução estão presentes na superfície do silício para todos os metais e aparentemente a relação entre as concentrações superficiais de cloreto e metal não segue a estequiometria original do cloreto metálico utilizado como fonte de contaminação intencional a não ser no caso do cobre onde a relação estequiométrica se verifica. Por outro lado, é interessante observar que as concentrações de $\mathrm{Ca}, \mathrm{Fe}, \mathrm{Ni}$, $\mathrm{Co}$ e $\mathrm{Cu}$ são da mesma ordem de grandeza. Em contraste, as concentrações superficiais de todos os metais foram certamente maiores do que a do cloreto cujo limite de detecção é de $1 \times 10^{13}$ átomos $/ \mathrm{cm}^{2}$. Estes resultados estão de acordo com aqueles reportados por M. Hourai et al. ${ }^{(18)}$ que descreveram a contaminação metálica de superfícies de lâminas de silício hidrofílicas. 
Tabela 1: Concentração superficial de contaminantes após a imersão em ADIF ou D-HF, onde ADIF e D-HF foram contaminados com 500 ppm de cálcio, ferro, níquel, cobalto ou cobre.

\begin{tabular}{|c|c|c|c|c|}
\hline Cloretos & \multicolumn{2}{|c|}{ Imersão em ADIFc (120 s) } & \multicolumn{2}{|c|}{ Imersão em D-HF (120 s) } \\
\hline 500 ppm de metal & Metal (átomos $/ \mathrm{cm}^{2}$ ) & Cloreto (Cl) (átomos $\left./ \mathrm{cm}^{2}\right)$ & Metal (átomos $/ \mathrm{cm}^{2}$ ) & Cloreto $(\mathrm{Cl})$ (átomos $/ \mathrm{cm}^{2}$ ) \\
\hline $\mathrm{CaCl}_{2}$ & $(6,8 \pm 1,7) 10^{14}$ & $(4,6 \pm 3,7) 10^{14}$ & $(4 \pm 4) 10^{13}$ & $<$ LD \\
\hline $\mathrm{FeCl}_{2}$ & $(4,0 \pm 1,0) 10^{14}$ & $(3,8 \pm 3,5) 10^{14}$ & $(6,8 \pm 2,5) 10^{13}$ & $(1,0 \pm 1,0) 10^{13}$ \\
\hline $\mathrm{NiCl}_{2}$ & $(1,0 \pm 0,2) 10^{15}$ & $(5,9 \pm 4) 10^{14}$ & $<\mathrm{LD}$ & $<$ LD \\
\hline $\mathrm{CoCl}_{2}$ & $(1,1 \pm 0,8) 10^{15}$ & $(8,3 \pm 5,0) 10^{14}$ & $(3 \pm 3) 10^{13}$ & $<$ LD \\
\hline $\mathrm{CuCl}_{2}$ & $(4,8 \pm 1,7) 10^{14}$ & $(9,6 \pm 2,4) 10^{14}$ & $>1 \times 10^{17}$ & $<$ LD \\
\hline
\end{tabular}

LD: Limite de detecção para TXRF.

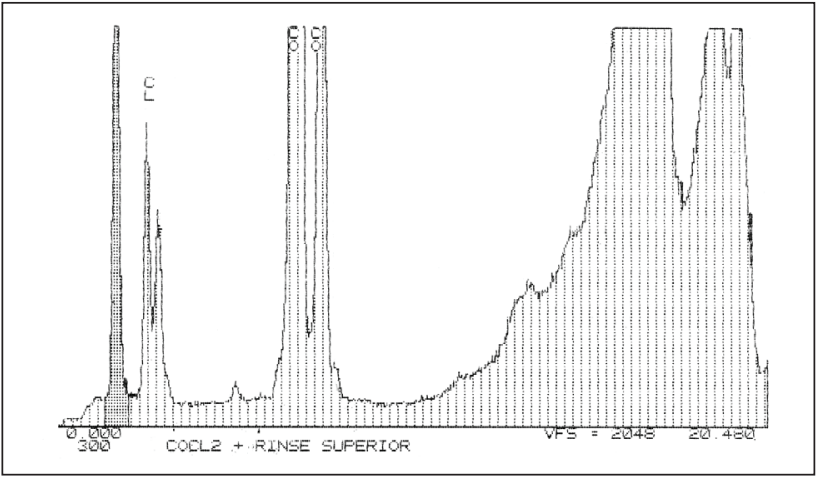

Figura 1: Espectro típico TXRF da lâmina de silício imersa em ADIF contendo 500 ppm de $\mathrm{CoCl}_{2}$.

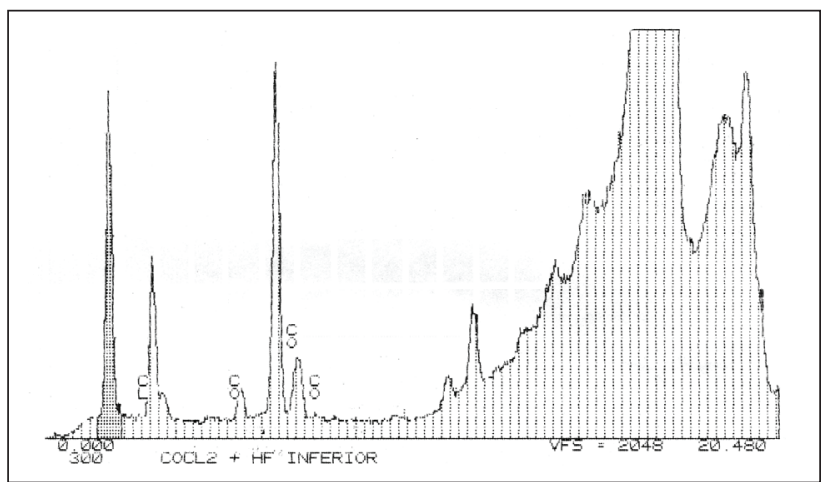

Figura 2: Espectro típico TXRF da lâmina de silício imersa em D-HF contendo 500 ppm de $\mathrm{CoCl}_{2}$.

Pode-se observar também na Tabela 1 três comportamentos diferentes para as lâminas que foram imersas em solução ácida (D-HF):

a. para o caso de Fe, valores mensuráveis de concentração de cloreto foram sempre encontrados na superfície do silício, enquanto a concentração do ferro correspondente permaneceu da mesma ordem de grandeza;

b. para os casos de $\mathrm{Ca}, \mathrm{Ni}$ e $\mathrm{Co}$, as concentrações de cloreto ficaram sempre abaixo do seu limite de detecção.

c. para o caso específico de $\mathrm{Cu}$, ocorre deposição e o nível de cloreto fica abaixo do limite de detecção.

Um modelo qualitativo pode ser proposto a partir destes resultados. O primeiro tipo de comportamento pode ser atribuído ao fato do $\mathrm{Fe}$ se complexar com o cloreto na forma $\mathrm{FeCl}_{2}$ em concentração que justificaria a sua presença na superfície aproximadamente no mesmo nível do Ferro ${ }^{(19)}$. Mecanismo semelhante é observado na lixiviação de minérios $^{(34)}$ enquanto o mesmo comportamento com o fluoreto é menos provável ${ }^{(35)}$. Já para os outros metais, como a complexação é mais difícil, o cloreto fica principalmente solvatado na solução. Assim, é extremamente significativo verificar que todos os metais estão na superfície com exceção do níquel. Era de se esperar que não houvesse cálcio na superfície devido ao seu alto potencial padrão de redução negativo, no entanto, o cálcio pode reagir com o fluoreto da superfície e permanecer na forma de $\mathrm{CaF}_{2}{ }^{(19)}$. No caso do cobalto, a sua complexação com cloreto pode dificultar sua presença na superfície em pH baixos ${ }^{(36,37)}$. Já no caso do cobre, a deposição está ocorrendo devido ao fato de seu potencial padrão de redução ser positivo.

Portanto, no caso de cloretos metálicos em solução D-HF, devemos analisar caso a caso a possibilidade de haver ou não complexação/precipitação do metal com cloreto ou fluoreto além de aplicar as regras eletroquímicas. No caso dos sulfatos metálicos, os mecanismos de contaminação são substancialmente mais simples uma vez que o fenômeno de complexação envolvendo sulfatos ocorre em menor grau comparado com os cloretos.

\section{CONCLUSÕES}

Analisamos a contaminação metálica superficial após imersão em solução diluída de ácido fluorídrico (D-HF) intencionalmente contaminada com cloretos metálicos. Os principais efeitos observados foram a complexação do Ferro com o cloreto resultando na presença de cloreto na superfície, o efeito de deposição espontânea de cobre que chega a formar um filme e a presença em níveis residuais de Ca e Co, provavelmente também associados a reações de complexação/precipitação com fluoreto.

\section{REFERÊNCIAS}

1. BUONASSISI, T.; HEUER, M.; ISTRATOV, A.A.; PICKETT, M.D.; MARCUS, M.A.; LAI, B.; CAI Z.; HEALD, S.M.; WEBER, E.R.; Transition metal co-precipitation mechanisms in silicon Retrive in https://publications.Ibl.gov/islandora/.../datastream/PDF/.../ citation...

2. ZOTH, G.; BERGHOLZ, W.; A Fast, preparation-free method to defect iron in silicon, Siemens AG, Components Group, Semiconductor Division, Otto-Hahn-Ring 6, 800 Munich 83, Federal Republic of Germany. ( Feb 1990) 
3. SHIMONO, T.; MORITA, M.; MURAMATSU Y.: TSUJI, M.; In Proceedings of the 8th Workshop on ULSI Ultra Clean Technology, Tokyo, 1990, p. 59.

4. TSUTIYA, N.; TANAKA, M.; KAGEYAMA, M.; KUBOTA, A.; MATSUSHITA, Y.; Extended Abstracts of the 22nd Conference on Solid State Devices and Materials, 1990, p. 1131.

5. JUN P. K.; LIM, H. B.; Study on the determination of contamination by Cu particles on silicon wafer surfaces. Anal. At. Spectrom., 18, 111-114 (2003)

6. JOLY, J.P.; Metallic contamination assessment of Silicon wafers Microelectronic Engineering 40 (1998) p. 285-294

7. DLAMINI, M.D.; Solar Energy Mater. Solar Cells 43, 353 (1996)

8. REEHAL, H.S.; LESNIAK, M.P.; HUGHES, A.E.; J. Phys. D: Appl. Phys. 29, 934 (1996)

9. ISTRATOV, A.A.; WEBER, E.R.; Electrical properties and recombination activity of copper, nickel and cobalt in silicon, Appl. Phys. A, 66(2), 123-136, 1998.

10. WEBER, E.R., In Properties of Silicon, EMIS Data reviews series No.4 (INSPEC, The Institution of Electrical Engineers, London, New York (1988) p. 409

11. HONDA, K.; OHSAWA, A.; TOYOKURA, N., Appl. Phys. Lett. 45, 270 (1984)

12. HIRAMOTO, K.; SANO, M.; SADAMITSU, S.; FUJINO, N., Jpn. J. Appl. Phys. Part 2 28, L2109 (1989)

13. REISS, J.H.; KING, R.R.; MITCHELL, K.W.; Appl. Phys. Lett. 68, $3302(1996)$

14. YOSHIDA, M.; FURUSHO, K., Behavior of Niquel as an Impurity in Silicon, Eletrotecnical Communication Laboratory, Midoricho, Musashinoshi, Tokio June 9, 1964

15. STRELI, C., Recent Advances in TXRF, Appl. Spectrosc. Rev., 41(5), 473-489 (2006).

16. EICHINGER, P.; RATH, H.J.; SCHWENKE, H.S., Application of total reflection $\mathrm{X}$-ray fluorescence analysis for metallic trace impurities on silicon wafer surfaces. In: Semiconductor fabrication: Technology and Metrology, ASTM STP990, Ed. Gupta, D.C. p. 305, 1988 (American Soc. for Testing and Materials).

17. HACKL, B.; et al., Correlation between DLTS and TRXFA measurements of copper and iron contaminations in $\mathrm{FZ}$ and CZ silicon wafers; application to gettering efficiences. J. Electrochem. Soc., v. 139, n. 5, p. 1495, 1992.

18. HOURAI, M.; NARIDOMI, T.; OKA, Y.; MURAKAMi, K.; SUMITA, S.; FUJINO, N.S., Jpn. J. Appl. Phys., v. 27, p. L2361, 1988.

19. LURIE, J., Handbook of Analytical Chemistry. Moscou, Mir Publishers, 1975. p. 76.

20. SANTOS FILHO, S. G., "Oxidação térmica rápida do silício: influência dos procedimentos de limpeza e dos perfis temporais de temperatura na qualidade dos óxidos de porta MOS". Tese de doutorado apresentada à Escola Politécnica da USP, Outubro de 1996.
21. SCHEMMEL, E., X-Ray Surface Analysis - a new method for measuring wafer surface contamination up to $10^{11}$ atoms $/ \mathrm{cm}^{2}$. Presented at conference: "New Manufacturing Structures and New Production Equipment for Semiconductor Production Lines" during Productronica, München 12-13 November, 1987.

22. HONDA, K.; A. OHSAWA, A.; TOYOKURA, N., J.Appl. Phys. 62 , 1960 (1987).

23. KITAGAWA, H.; TANAKA, S.; NAKASHIMA, H.; YOSHIDA, M., J. Electron. Mater. 20, 441 (1991)

24. KITAGAWA, H.; NAKASHIMA, H., Jpn. J. Appl. Phys. 28, 305 (1989)

25. KITAGAWA, H.; NAKASHIMA, H., Phys. Status Solidi (A) 99, K49 (1987)

26. KITAGAWA, H.; NAKASHIMA, H., Phys. Status Solidi (A) 102, K23 (1987)

27. CHUA, W.B.; ROSE, K., J. Appl. Phys. 41, 2644 (1970)

28. SHABANI, M.B.; YOSHIMI, T.; OKUUCHI, S.; Abe, H., Solid State Phenom. 57-58, 81 (1997)

29. MORINAGA, $H_{\text {.; }}$ SUYAMA, M.; NOSE, M.; VERHAVERBEKE, S.; OHMI, T., in Proceedings of Second International Symposium on UCPSS, p.217, Bruges, September (1994).

30. MORINAGA, H.; SUYAMA, M.; NOSE, M.; VERHAVERBEKE, S.; OHMI, T., IEICE Trans., 1996, E79-C, 343.

31. SHIMONO, T.; TSUJI, M., In Extended Abstracts of Electrochemical Society, Washington, DC, 1991, 91(1), p. 278.

32. HOFF, A. M.; ARAVAMUDHAN, S.; ISTI, A.; E. I. OBORINA, E. I., Degradation of Oxide Properties Caused by Low-Level Metallic Contamination. J. Electrochem. Soc., v. 154, n. 11, p. H977-H982, 2007

33. KHALIL, R.; KVEDER, V.; SCHRÖTER, W.; SEIBT, M., Early stages of iron precipitation in silicon. phys. stat. sol. (c) 2, No. 6, 18021806 (2005) / DOI 10.1002/pssc.200460507

34. MCDONALD, R.G.; WHITTINGTON, B.I., Atmospheric acid leaching of nickel laterites review: Part I. Sulphuric acid technologies, Hydrometallurgy, Volume 91, Issues 1-4, March 2008, Pages 35-55, ISSN 0304-386X, http://dx.doi.org/10.1016/j. hydromet.2007.11.009.

35. SOLI, A.L.; BYRNE,R. H., The hydrolysis and fluoride complexation behavior of $\mathrm{Fe}(\mathrm{III})$ at $25^{\circ} \mathrm{C}$ and 0.68 molal ionic strength, Journal of Solution Chemistry Volume 25, Issue 8, pp 773-785 (1996)

36. FANG, J.; LI, M.; XU,Z., Separation of Cobalt from a NickelHydrometallurgical Effluent Using an Emulsion Liquid Membrane, Separation Science and Technology, Volume 38, Issue 14, pages 3553-3574, 2003, DOI: 10.1081/SS-120023417

37. KIM, H.; BAEK, K.; KIM, B.; YANG, J.; Humic substance-enhanced ultrafiltration for removal of cobalt, Journal of Hazardous Materials, Volume 122, Issues 1-2, 30 June 2005, Pages 31-36, ISSN 03043894, http://dx.doi.org/10.1016/j.jhazmat.2005.03.043. 